\title{
IDENTIFICAÇÃO DOS PRINCIPAIS PARASITOS INTESTINAIS ENCONTRADOS EM LOCAIS PÚBLICOS NO BRASIL
}

\section{ARTIGO DE REVISÃO}

GUERRA, Matteus Teixeira ${ }^{1}$

LIMA, Jonas Hantt Corrêa ${ }^{2}$

TESSARO, Julia Carolina Lusa ${ }^{3}$

SILVA, Paulo Henrique ${ }^{4}$

FACHINI, Jean ${ }^{5}$

KOBER, Márcia de Vargas ${ }^{6}$

GUERRA, Matteus Teixeira. Et al. Identificação dos principais parasitos intestinais encontrados em locais públicos no Brasil. Revista Científica Multidisciplinar Núcleo do Conhecimento. Ano 05, Ed. 10, Vol. 15, pp. 121-137. Outubro de 2020. ISSN: 2448-0959, Link de acesso: https://www.nucleodoconhecimento.com.br/saude/principais-parasitos

\section{RESUMO}

As infecções causadas por parasitos são consideradas uma das maiores causas de agravos por processos infecciosos no mundo, e, segundo estimativa, afetam cerca de

\footnotetext{
${ }^{1}$ Bacharel em Biomedicina - ULBRA - Canoas/RS.

${ }^{2}$ Acadêmico de Medicina - ULBRA - Canoas/RS.

${ }^{3}$ Acadêmica de Medicina - ULBRA - Canoas/RS.

${ }^{4}$ Acadêmico de Medicina - ULBRA - Canoas/RS.

${ }^{5}$ Acadêmico de Biomedicina - ULBRA - Canoas/RS.

${ }^{6}$ Orientadora. Doutora, Bióloga.
} 
2 bilhões de pessoas em todo os continentes, tornando-se um dos principais problemas de saúde pública principalmente no Brasil. Esses parasitos eliminam seus ovos, larvas ou cistos junto com as fezes do hospedeiro e contaminam o ambiente, o solo e a água, além do contato das mãos sujas levadas à boca podendo ocasionar infecções parasitárias. Esses e outros fatores, somados às condições climáticas do Brasil e a falta de saneamento básico são importantes processos para disseminação desses parasitos no país. Vários estudos demonstraram que locais públicos podem atuar como reservatórios para parasitos intestinais, de acordo com eles foram selecionados os parasitos mais prevalentes: Entamoeba coli, Entamoeba histolytica, Giardia intestinalis, Endolimax nana, Ascaris lumbricoides, Enterobius vermicularis, Strongyloides stercolaris, Toxocara spp e ancilostomídeos. O estudo identificou os três principais parasitos intestinais entre todos os 19 artigos analisados e encontrou 13 espécies de parasitos intestinais nos diferentes locais pesquisados pelos autores. O presente estudo demonstrou, através da análise de 19 artigos, que os locais públicos no Brasil podem atuar como reservatórios para parasitos intestinais, e as pessoas que frequentam esses locais constituem um grupo de risco estando sujeitas às infecções parasitárias.

Palavras-chave: Parasitos intestinais, locais públicos, prevalência, helmintos.

\section{INTRODUÇÃO}

As infecções causadas por parasitos são consideradas uma das maiores causas de agravos por processos infecciosos no mundo, e, segundo estimativa, afetam cerca de 2 bilhões de pessoas em todo os continentes, tornando-se um dos principais problemas de saúde pública principalmente no Brasil. Estas parasitoses podem ter diversas manifestações clínicas como desnutrição, anemia, deficiência intelectual, irritabilidade e diarreia, além disso os indivíduos ficam mais suscetíveis a contrair outras infecções (MENEZES et al., 2012; COSTA et al., 2018).

Em uma escala global, o número de infecções confirmadas por parasitos é alto. Estima-se que 1,5 bilhão de indivíduos esteja infectado com Ascaris lumbricoides, 1,3 bilhão com Trichuris trichiura, 1,05 bilhão com ancilostomídeos, 200 milhões com 
Entamoeba histolytica e 400 milhões com Giardia duodenalis. No continente americano o Ascaris lumbricoides é o parasito mais prevalente, afetando cerca de 30\% da população (ANDRADE et al., 2008; CURVAL et al., 2017).

Os parasitos eliminam seus ovos, larvas ou cistos junto com as fezes do hospedeiro, contaminando o ambiente e o solo, podendo os ovos e os cistos serem levados pela poeira aos alimentos ou arrastados por correntes de água. A água pode ser contaminada através de enxurradas que atingem mananciais utilizados para abastecimento de cidades e irrigação de plantações. $O$ contato das mãos sujas levadas diretamente à boca também pode oferecer riscos de contaminação. As larvas, por sua vez, podem penetrar ativamente através da pele (SIQUEIRA e PEDROSO, 1997).

No Brasil devido às condições climáticas, a falta de políticas públicas que atuem de forma efetiva na área da saúde, fatores socioeconômicos e comportamentais, a falta de saneamento e acesso limitado à água potável desempenham um papel importante para a manutenção e proliferação de focos endêmicos destes parasitos (CURVAL et al., 2017; ASSIS et al., 2018).

Segundo estudos, o processo de transmissão de alguns parasitos como os helmintos é amplificado pela resistência dos ovos a agentes terapêuticos, detergentes e desinfetantes de uso doméstico e à radiação ultravioleta (UV). Devido a essa resistência acentuada dos ovos, os ambientes públicos de grande circulação de pessoas representam alto potencial de transmissão destes agentes patogênicos, pois as pessoas estão em constante e múltiplo contato com diversas partes destes locais (SCHOLTE et al., 2015; MURTA e MASSARA, 2015).

Locais públicos recebem pessoas de todas as classes sociais, nem todos têm saneamento básico e acesso à água potável. Devido a esse alto fluxo de pessoas, esses locais podem se tornar reservatórios para parasitos intestinais (ASSIS et al., 2018). 
Tendo em vista as diversas formas de disseminação dos parasitos já descritos, sua facilidade de transmissão, prolongada resistência e considerando a importância do problema das parasitoses no Brasil, seus mecanismos de transmissão e o menosprezo pelas autoridades de saúde no país, se faz necessário uma análise dos principais parasitos intestinais em locais públicos no Brasil.

Este estudo se propõe a realizar um levantamento parasitológico através de uma revisão bibliográfica, identificando os principais parasitos intestinais presentes em locais públicos no Brasil, e apresentando os dados em forma de porcentagem de acordo com a prevalência nos artigos analisados.

Diversos estudos já descreveram locais que atuam como carreadores de parasitos intestinais: cédulas de dinheiro (COSTA et al., 2018); terminais aeroportuários (SCHOLTE et al., 2015); ônibus de transporte público (ASSIS et al., 2018); sanitários de pré-escolas (COELHO et al., 1999); salas de espera e sanitários de Unidades Básicas de Saúde (UBS) (SILVA et al., 2013); chupetas de crianças (SIQUEIRA e PEDROSO, 1997) e hortaliças em feiras livres (GUILHERME et al., 1999). Analisando diversos trabalhos que relatam a presença destes organismos, serão apresentados os principais parasitos intestinais identificados com maior frequência em locais públicos no Brasil.

\section{ENTAMOEBA COLI}

A Entamoeba coli é um protozoário geralmente não patogênico presente no intestino grosso dos humanos. Sua locomoção e alimentação é feita através de pseudópodes. Esse protozoário pode ser encontrado na forma de cisto ou trofozoíto. As duas formas podem ser encontradas nas fezes. Os cistos são esféricos e ovoides, contêm uma parede cística espessa, conforme o grau de desenvolvimento, contêm de quatro a oito núcleos. À medida que o número de núcleos aumenta o diâmetro nuclear e a quantidade de cromatina do cisto reduzem (NEVES, 2016; SMITH, 2017). 


\section{ENTAMOEBA HISTOL YTICA/DISPAR}

A Entamoeba histolytica é o parasito causador da amebíase, seu ciclo é monoxeno, ou seja, completa em apenas um hospedeiro, e seu modo de infecção é fecal/oral. Após a ingesta de cistos, os trofozoítos colonizam o lúmen intestinal e se multiplicam e vivem como comensais, usando bactérias e restos celulares como fonte de energia. Quando os trofozoítos tornam-se patogênicos e invadem a parede intestinal, alimentam-se de células da mucosa e de hemácias que gera uma reação inflamatória levando a destruição do tecido envolvido (SANTOS e SOARES, 2008).

Devido à semelhança existente entre os cistos de Entamoeba histolytica e Entamoeba coli é preciso fazer um diagnóstico diferencial através da morfologia e do número de núcleos. Os cistos de Entamoeba histolytica podem ter de 1 a 4 núcleos e os cistos de Entamoeba coli possuem de 4 a 8 núcleos (SANTOS e SOARES, 2008; REY, 2010; NEVES, 2016).

A Entamoeba dispar é considerada não patogênica, porém os únicos modos de fazer a diferenciação dessas duas espécies morfologicamente idênticas é através do imunoensaio enzimático ELISA, que detecta antígenos específicos para E. histolytica, ou através de testes de biologia molecular como o PCR (TOMÉ e TAVARES, 2007; REY, 2010).

\section{GIARDIA INTESTINALIS}

A Giardia intestinalis é um parasito que pode se apresentar tanto na forma de cisto quanto trofozoíto e suas duas formas podem ser encontradas nas fezes. É mais comum encontrar em fezes diarreicas a forma de trofozoítos, e nas fezes sólidas são encontrados os cistos (PEDROSO e AMARANTE, 2006; SANTANA et al., 2014).

Os cistos de Giardia intestinalis constituem a sua forma infectante e dentro deste cisto o núcleo se duplica, com isso quando o homem ingere um cisto ele se infecta com dois trofozoítos, porém tanto os cistos quanto os trofozoítos podem ser ingeridos pelo homem através da água ou alimentos contaminados. O desencistamento ocorre 
através da ação de enzimas digestivas dando origem aos trofozoítos, que podem ficar na luz intestinal ou se fixarem na parede do duodeno. Se ocorrer do parasito aderir à mucosa intestinal a absorção de nutrientes do infectado fica comprometida, principalmente de gorduras e de vitaminas lipossolúveis. A sua prevalência é predominante nas crianças entre zero e seis anos, pois existe o contato interpessoal em creches e piscinas comunitárias, ocorrendo uma maior disseminação desse parasito devido às condições sanitárias precárias. A maioria dos casos é assintomática, principalmente em pacientes adultos. Em crianças e jovens podem apresentar amplo aspecto clínico, podendo evoluir para quadros de hemorragia retal e fenômenos alérgicos (PEDROSO e AMARANTE, 2006; SANTANA et al., 2014).

\section{ENDOLIMAX NANA}

É a menor das amebas intestinais que infectam humanos, tendo seu trofozoíto em torno de $8 \mu \mathrm{m}$. O trofozoíto vive no intestino grosso do hospedeiro e é geralmente considerado não patogênico. De acordo com alguns estudos, a prevalência em algumas populações pode chegar a $30 \%$. O seu ciclo de vida é idêntico ao de outras amebas, formando cistos e sendo esse o estágio infeccioso (POULSEN e STENSVOLD, 2016).

Os cistos de Endolimax nana podem ser distinguidos das outras amebas por seu tamanho menor, tendo 9 um o maior diâmetro do seu cisto. Os trofozoítos alimentamse ativamente de bactérias e multiplicam-se rapidamente através da clivagem binária. Esses parasitos vivem no cólon e também podem ser encontrados no apêndice, movem-se através de pseudopodia e podem atingir um tamanho de até $30 \mu \mathrm{m}$ durante a atividade motora. Uma infecção causada por Endolimax nana pode durar vários anos de forma assintomática (POULSEN e STENSVOLD, 2016).

\section{ASCARIS LUMBRICOIDES}

É o maior nematoide que vive no intestino delgado humano, seus ovos apresentam em média $60 \mu \mathrm{m}$ e podem ser de dois tipos: fertilizados e não fertilizados. Os ovos fertilizados têm uma forma oval e uma casca de três camadas grossas. A camada 
mais externa é uma membrana mamilonada formada por mucopolissacarídeos, abaixo dela está a camada semipermeável formada por quitina e proteínas, na camada interna existe uma membrana vitelina não permeável constituída por proteínas e lipídios. Essa estrutura formada por camadas permite o ovo ter uma grande durabilidade contra diversos fatores químicos e ambientais, sendo um dos ovos mais resistentes entre os parasitos intestinais, podendo ser preservados por até 40 dias em temperaturas entre 18 a $27^{\circ} \mathrm{C}$ (DOLD e HOLLAND, 2011; BEYHAN et al., 2016).

Os hospedeiros contraem a infecção por Ascaris lumbricoides através da rota fecal/oral, após os ovos infectados serem ingeridos e eclodirem na região intestinal, as larvas se desenvolvem nos tecidos parentéricos e podem migrar através dos vasos ou peritônio até os pulmões. Após a chegada nos pulmões as larvas migram até a orofaringe e retornam ao intestino, tornando-se sexualmente maduros para ocorrer o acasalamento e a ovipostura (DOLD e HOLLAND, 2011; BEYHAN et al., 2016).

Este parasito é a espécie mais conhecida entre os nematoides por ser extremamente comum na espécie humana. Ainda hoje é responsável por causar grandes problemas de saúde pública, especialmente nos países em desenvolvimento, de uma forma geral as crianças são as mais atingidas e apresentam os sinais clínicos mais relevantes na infecção parasitária (CAMPOS et al., 2002).

\section{ENTEROBIUS VERMICULARIS}

É um helminto nematóide que mede cerca de 0,3 a $1,0 \mathrm{~cm}$ e é conhecido como Oxiúro. $\mathrm{Na}$ sua forma morfológica apresenta-se na forma adulta com asas cefálicas. Após o acasalamento, o macho é eliminado nas fezes e a fêmea adulta se dirige até o ânus, ocorrendo a ovipostura, principalmente à noite. É comum a fêmea adulta não retornar para a ampola retal, morrendo no local (COOK, 1994).

Os ovos maturam rapidamente na região perianal ou no solo apresentando larvas infectantes, esses ovos maduros devem ser ingeridos pelo hospedeiro para a continuidade do ciclo, após a ingesta os ovos eclodem no intestino delgado e as larvas migram pela mucosa intestinal até o ceco atingindo a maturidade. Esta espécie pode 
provocar lesões significativas na mucosa, e na região perianal pode haver laceração da pele com hemorragia e outras infecções secundárias (COOK, 1994).

\section{STRONGYLOIDES STERCOLARIS}

É o menor dos nematoides que parasitam o homem em nosso meio, medem cerca de 2 a $3 \mathrm{~mm}$. Seus ovos são eliminados nas fezes da pessoa contaminada, a eclosão e liberação são extremamente rápidas. Este parasito é uma das exceções que pode gerar uma autoinfecção endógena, podendo resultar em uma infecção duradoura. Alguns estudos relatam infecções mantidas por cerca de 75 anos (SCHÄR et al., 2013; NEVES, 2016).

As larvas fazem a penetração da mucosa intestinal e em seguida iniciam um ciclo até o duodeno (autoinfecção), a única forma adulta é a fêmea. As larvas presentes no solo podem infectar o homem através da penetração cutânea levando a inflamação no local e hipersensibilidade após várias infecções (SCHÄR et al., 2013; NEVES, 2016).

\section{ANCILOSTOMÍDEOS}

A família dos Ancilostomídeos constitui uma das mais importantes entre os nematóides. Esses parasitos são conhecidos por desencadearem um importante processo patológico crônico, podendo em alguns casos levar a morte (NEVES, 2016).

Existem dezenas de espécies descritas desses parasitos, porém Ancylostoma duodenale e Necator americanus sãos os mais importantes agentes etiológicos quando falamos da ancilostomose humana (CORREA et al., 1979; VALENTE, 2013).

Suas larvas variam de $0,8 \mathrm{~mm}$ a $1,3 \mathrm{~cm}$ e quando eliminados nas fezes são avermelhados pela hematofagia e histiofagia que causam no trato gastrointestinal do hospedeiro. Os ovos desses parasitos são depositados pela fêmea no intestino delgado e depois liberados no ambiente através das fezes para tornarem-se larvados. Não é possível a diferenciação de ovos desses parasitos. Após eclodir a larva rabtoide leva em torno de uma semana para tornar-se filarióide, sendo essa a que penetra na pele do homem ocasionando nas infecções. Ao chegar ao intestino delgado os 
ancilostomídeos exercem o parasitismo hematófago, fixando a cápsula bucal na mucosa do duodeno, e a diferenciação para a larva adulta ocorre em torno de 30 dias após a infecção (CORREA et al., 1979).

\section{TOXOCARA SPP}

Toxocara spp, em seus estágios larvais, por migração errática, ocasionam uma importante zoonose. É considerado um dos parasitos mundiais mais comuns, sendo - Toxocara canis o de maior prevalência entre eles, podendo chegar até $81 \%$ da população de cães (SANTARÉM et al., 1998).

A infecção em humanos é mais frequente em crianças com idade entre um e cinco anos, cujos hábitos levam-nas a ingerirem ovos embrionados de Toxocara spp que muitas vezes se encontram em bancos de areias e parques recreativos. Estas larvas eclodirão no intestino e migrarão pela via linfática ou circulação portal para diversos órgãos, principalmente fígados e pulmões, em alguns casos podem migrar para o coração e sistema nervoso, causando a síndrome Larva migrans visceral, ou afetando o globo ocular e gerando a síndrome Larva migrans ocular (SANTARÉM et al., 1998).

\section{TAENIA SOLIUM}

É um cestódeo que possui ventosas, ganchos no escólece e cerca de um metro e meio de comprimento, podendo atingir de cinco a seis metros. Esse parasito é o causador da teníase, uma parasitose intestinal causada pela ingestão de cisticercos; o verme adulto pode provocar no intestino humano um aumento da motilidade intestinal e inflamação crônica (ISAAC et al., 2019).

Além da teníase, a Taenia solium também é causadora da cisticercose, que constitui no desenvolvimento de cisticercos no tecido humano, decorrentes da ingestão acidental de ovos de Taenia. Ocorre em vários locais do organismo, principalmente no cérebro (causando neurocisticercose), olhos, músculos e fígado (WHITE-JÚNIOR et al., 2018). 


\section{TAENIA SAGINATA}

É um cestódeo causador da teníase, diferente da Taenia solium, não possui ganchos no escólece, apenas ventosas. Possui mais de 1 metro e meio de comprimento e pode atingir até 12 metros. Há eliminação de proglotes nas fezes da pessoa contaminada, cada um possuindo 20 a 70 mil ovos. O hospedeiro intermediário (boi) ingere os ovos e desenvolve o cisticerco nos músculos. O homem pode ingerir a carne contaminada e mal cozida, levando ao desenvolvimento da Taenia saginata adulta no intestino (WHITE-JÚNIOR et al., 2018; ISAAC et al., 2019).

\section{DESENVOLVIMENTO}

Para realização deste estudo foi feito uma revisão bibliográfica sobre o tema nas revistas científicas disponíveis online, por meio dos portais de pesquisa científica PubMed e Google Acadêmico. Foram reunidos e comparados os diferentes dados encontrados nas fontes consultadas e identificados os principais parasitos intestinais em locais públicos no Brasil.

Os descritores selecionados e suas respectivas combinações foram: parasitos, helmintos, parasitos intestinais, prevalência de parasitos intestinais, parasitos intestinais em locais públicos. Nas bases internacionais, adicionou-se o termo "Brasil" nos descritores mencionados, a fim de filtrar trabalhos aplicados no país, para se adequar ao objetivo do trabalho.

Não foi definido um intervalo entre anos para a seleção de pesquisas, nem se limitou pelo idioma escrito. Os artigos publicados em outros idiomas poderiam ser utilizados desde que se tratasse de pesquisas realizadas em território nacional.

Os critérios de inclusão se deram pela necessidade do trabalho em retratar sobre parasitos intestinais em locais públicos no Brasil. O critério de exclusão se deu pela análise dos parasitos em amostras biológicas.

Durante as análises foram selecionados os três parasitos intestinais mais prevalentes de cada estudo e seu local de coleta. 


\section{RESULTADOS E DISCUSSÃO}

Foram analisados 19 artigos que se adequaram aos requisitos de pesquisa. Destes, 18 apresentavam resultados positivos para a presença de parasitos intestinais e apenas 1 não registrou a presença de parasitos.

Nestes trabalhos foram identificados a presença de 13 espécies de parasitos entre os três mais prevalentes de cada artigo que estão representados no Quadro 1.

Quadro 1. Listagem dos três principais parasitos intestinais de cada artigo e suas porcentagens de positividade, local de coleta e autor.

\begin{tabular}{|c|c|c|}
\hline Autor/Ano & Local & Parasitos/Porcentagens \\
\hline Assis et al. (2018) & Transporte público. & $\begin{array}{l}\text { Enterobius vermicularis (7,5\%) } \\
\text { Hymenolepis sp. (4,2\%) } \\
\text { Ascaris lumbricoides (3,3\%) }\end{array}$ \\
\hline $\begin{array}{l}\text { Gomes et al. } \\
(2016)\end{array}$ & Transporte público. & Giardia intestinalis $(1,6 \%)$ \\
\hline $\begin{array}{l}\text { Scholte et al. } \\
(2015)\end{array}$ & $\begin{array}{l}\text { Terminal } \\
\text { aeroportuário. }\end{array}$ & $\begin{array}{l}\text { Não houve amostras positivos para } \\
\text { parasitos intestinais neste local. }\end{array}$ \\
\hline Santos (2015) & Transporte público. & Ascaris lumbricoides (16,3\%) \\
\hline $\begin{array}{l}\text { Nomura et al. } \\
(2015)\end{array}$ & Feira livre. & $\begin{array}{l}\text { Endolimax nana (100\%) } \\
\text { Ancilostomideos (87,5\%) } \\
\text { Balantidium coli e Entamoeba } \\
\text { histolytica/díspar (87,5\%) }\end{array}$ \\
\hline $\begin{array}{l}\text { Murta \& Massara. } \\
(2015)\end{array}$ & Transporte público. & $\begin{array}{l}\text { Hymenolepis sp (93,3\%) } \\
\text { Enterobius vermicularis (56,7\%) } \\
\text { Ascaris lumbricoides }(23,3 \%)\end{array}$ \\
\hline
\end{tabular}




\begin{tabular}{|c|c|c|}
\hline $\begin{array}{l}\text { Damázio et al. } \\
(2015)\end{array}$ & Papel-moeda. & $\begin{array}{l}\text { Giardia intestinalis }(2,22 \%) \\
\text { Entamoeba coli }(1,48 \%) \\
\text { Ancilostomídeos }(1,48 \%)\end{array}$ \\
\hline $\begin{array}{l}\text { Chen \& Mucci } \\
(2015)\end{array}$ & $\begin{array}{l}\text { Área de recreação } \\
\text { infantil. }\end{array}$ & $\begin{array}{l}\text { Ascaris lumbricoides (100\%) } \\
\text { Toxocara spp (100\%) } \\
\text { Ancilostomideos (50\%) }\end{array}$ \\
\hline $\begin{array}{l}\text { Valadares et al. } \\
(2014)\end{array}$ & Sanitários públicos. & $\begin{array}{l}\text { Entamoeba coli }(83,3 \%) \\
\text { Endolimax nana }(66,6 \%) \\
\text { lodamoeba butschlii }(58,3 \%)\end{array}$ \\
\hline Silva et al. (2013) & $\begin{array}{l}\text { Unidades básicas } \\
\text { de saúde. }\end{array}$ & Enterobius vermicularis $(13,5 \%)$ \\
\hline $\begin{array}{l}\text { Aguiar } \quad \& \\
\text { Lamounier. } \\
\text { (2013) }\end{array}$ & Papel-moeda. & $\begin{array}{l}\text { Endolimax nana }(42,10 \%) \\
\text { Entamoeba coli }(36,84 \%) \\
\text { lodamoeba butschlii }(10,52 \%)\end{array}$ \\
\hline $\begin{array}{l}\text { Fernandes et al. } \\
(2012)\end{array}$ & Transporte público. & $\begin{array}{l}\text { Entamoeba coli }(57,5 \%) \\
\text { Endolimax nana (10\%) } \\
\text { Entamoeba histolytica/Dispar }(2,5 \%)\end{array}$ \\
\hline Ross et al. (2011) & Praças públicas. & $\begin{array}{l}\text { Ancilostomideos (18\%) } \\
\text { Ascaris lumbricoides (6\%) } \\
\text { Trichuris sp (5\%) }\end{array}$ \\
\hline $\begin{array}{l}\text { Borges et al. } \\
(2009)\end{array}$ & $\begin{array}{l}\text { Sanitários e } \\
\text { transporte público. }\end{array}$ & $\begin{array}{l}\text { Parasitos em sanitários: } \\
\text { Ascaris lumbricoides (6,25\%) }\end{array}$ \\
\hline
\end{tabular}




\begin{tabular}{|c|c|c|}
\hline & & $\begin{array}{l}\text { Enterobius vermicularis }(6,25 \%) \\
\text { Parasitos em transporte público: } \\
\text { Enterobius vermicularis }(18,7 \%)\end{array}$ \\
\hline $\begin{array}{l}\text { Piccolo \& } \\
\text { Gagliani. (2008) }\end{array}$ & Papel-moeda. & $\begin{array}{l}\text { Ascaris lumbricoides }(11,6 \%) \\
\text { Entamoeba coli }(10,6 \%) \\
\text { Toxocara canis }(9,3 \%)\end{array}$ \\
\hline $\begin{array}{l}\text { Araújo et al. } \\
(2008)\end{array}$ & $\begin{array}{l}\text { Área de recreação } \\
\text { infantil. }\end{array}$ & $\begin{array}{l}\text { Ascaris lumbricoides (88\%) } \\
\text { Ancilostomideos (42\%) } \\
\text { Strongyloides sp (39\%) }\end{array}$ \\
\hline $\begin{array}{l}\text { Matesco et al. } \\
(2007)\end{array}$ & Praia. & $\begin{array}{l}\text { Ascaris lumbricoides (8,3\%) } \\
\text { Toxocara spp (3,3\%) } \\
\text { Ancilostomideos }(1,7 \%)\end{array}$ \\
\hline Brito et al. (2006) & Papel-moeda. & $\begin{array}{l}\text { Ascaris lumbricoides (22\%) } \\
\text { Balantidium coli } 18 \%\end{array}$ \\
\hline $\begin{array}{l}\text { Costa-Cruz et al. } \\
\text { (1995) }\end{array}$ & $\begin{array}{l}\text { Distribuidores de } \\
\text { alimentos. }\end{array}$ & $\begin{array}{l}\text { Giardia intestinalis }(21,1 \%) \\
\text { Entamoeba coli }(21,1 \%) \\
\text { Ancilostomideos }(9,6 \%)\end{array}$ \\
\hline
\end{tabular}

Considerando a presença de parasitos intestinais em locais públicos, os resultados do presente estudo relataram uma positividade de $95 \%$ perante os 19 artigos analisados, um resultado tão significativo se dá pelo potencial de transmissão desses parasitos, que podem ser transmitidos de uma pessoa para outra ou por objetos inanimados que atuam como fômites (SUDRÉ et al., 2012). 
O resultado do parasito mais encontrado durante as análises dos artigos corrobora com a prevalência mundial dos parasitos. A Ascaris lumbricoides foi a espécie mais encontrada entre todos os estudos, sendo o parasito mais prevalente no Brasil. Esse parasito esteve presente em vários locais, diferente de outros que são mais específicos como o Toxocara spp, que é tradicionalmente encontrado em ambientes com areia, pois se trata de um parasito que é transmitido através das fezes de cães e responsável por uma importante zoonose (MATESCO et al., 2007; CHEN e MUCCI, 2015).

A alta prevalência de Ascaris lumbricoides é justificada, pois se trata de um parasito que apresenta uma membrana externa que proporciona aderência a superfície, a preservação do ovo em temperaturas entre 18 a $27^{\circ} \mathrm{C}$, além dessa estrutura formada por camadas permitir o ovo ter uma grande durabilidade contra diversos fatores químicos, como detergentes comuns utilizados na limpeza doméstica (DOLD e HOLLAND, 2011; SANTOS, 2015; BEYHAN et al., 2016).

Os transportes públicos demonstraram-se como um importante reservatório de parasitos intestinais, sendo os mais encontrados nesse local: Ascaris lumbricoides e Entamoeba. Esses transportes recebem diversas pessoas, e que muitas vezes acabam não realizando a higienização das mãos, levando esses parasitos para dentro dos transportes públicos que, por consequência, irão atuar como fômites (FERNANDES et al., 2012; SANTOS, 2015; MURTA e MASSARA, 2015; GOMES et al., 2016; ASSIS et al., 2018).

Os ancilostomídeos foram identificados entre os mais prevalentes em diversos trabalhos analisados, mas assim como Toxocara spp se mantiveram em locais específicos. Foi possível identificar esses parasites, principalmente em locais com areia, mas também foram encontrados em cédulas de dinheiro, feiras de verduras e distribuidores de alimentos (COSTA-CRUZ et al., 1995; MATESCO et al., 2007; ARAÚJO et al., 2008, ROSS et al., 2011; NOMURA et al., 2015; CHEN e MUCCl, 2015). 
O único artigo analisado que não foi encontrado amostras parasitárias foi em um terminal aeroportuário. Para este local os autores citam que é cumprida as normas de limpeza e desinfecção da Agência Nacional de Vigilância Sanitária (ANVISA), sendo possível diminuir ou até mesmo anular a disseminação desses parasitos em ambientes com grande fluxo de pessoas (SCHOLTE et al., 2015).

Apesar de ter sido descrito na literatura, Taenia spp não foi encontrado entre os parasitos mais prevalentes nos artigos analisados, sendo detectado em apenas um trabalho em poucas amostras. Apesar de descrito sobre a resistência dos ovos de Taenia spp a fatores do ambiente, não foi encontrado relatos na literatura de resistência a fatores químicos, não atuando como os ovos de Ascaris lumbricoides (PICCOLO e GAGLIANI, 2008).

No Brasil poucos estudos foram feitos sobre a prevalência de parasitos intestinais em locais públicos que não utilizam amostras biológicas humanas ou de animais, um dos primeiros relatos foi o artigo publicado por Levai et al. (1986), os autores retrataram a prevalência de parasitos em cédulas de dinheiro na cidade de São Paulo. Tanto nos trabalhos seguintes a Levai et al. (1986) sobre papel-moeda, quanto para prevalência em outros locais e objetos, existe uma grande divergência quanto aos resultados de parasitos encontrados no Brasil, os motivos para essa diferença podem ser: prevalência de doenças parasitárias de cada região, diferenças climáticas, situação socioeconômica-sanitária, métodos de coleta, preparo e análise das amostras e também a superfície de coleta.

As pessoas que utilizam esses locais públicos constituem o grupo de risco para contaminação parasitária, pois estão sujeitas a ambientes fechados e múltiplos contatos com diversas superfícies que podem atuar como reservatório para parasitos intestinais, pois são locais com alto fluxo de pessoas de diferentes classes sociais, hábitos culturais e higiênicos. Para esse grupo de risco é imprescindível realizar a higienização das mãos, sendo um importante método de prevenção na transmissão de parasitos e diversos outros patógenos. Essa transmissão ocorre através da capacidade dos ovos ficarem abrigados na pele e transferi-los de uma superfície para outra, por contato direto ou indireto através de objetos (GOMES et al., 2016). 
Através da alta prevalência analisada nos resultados, é demonstrado a importância da higienização pessoal das mãos, dos locais públicos e da manutenção do saneamento básico para o controle epidemiológico de diversas doenças, incluindo as parasitoses.

\section{CONSIDERAÇÕES FINAIS}

O presente estudo demonstrou através da análise de 19 artigos que os locais públicos no Brasil podem atuar como reservatórios para parasitos intestinais, e as pessoas que frequentam esses locais constituem um grupo de risco estando sujeitas às infecções parasitárias.

Esses resultados demonstram uma necessidade de uma limpeza mais intensa nesses locais, além de demonstrar a importância da manutenção de higiene pessoal e do saneamento básico de cada cidade. Além disso, sugere-se que as pessoas que frequentam esses locais carreguem consigo álcool em gel para prevenir estas parasitoses e outras patologias que estão sujeitas.

\section{REFERÊNCIAS}

AGUIAR, Narayane Sales; LAMOUNIER, Thaís Alves da Costa. Pesquisa de cistos de protozoários e ovos de helmintos em cédulas de dinheiro na cidade de Gama-DF. Acta de Ciência e Saúde, v. 2, n. 2, p. 1-13, 2013.

ANDRADE, Elisabeth Campos de; LEITE, Isabel C. Gonçalves; VIEIRA, Marcel de T. Vieira; ABRAMO, Clarice; TIBIRIÇÁ, Sandra H. Cerrato; SILVA, Priscila Lima. Prevalência de parasitoses intestinais em comunidade quilombola no município de Bias Fortes, Estado de Minas Gerais, Brasil. Epidemiologia Serviço e Saúde, v. 20, n. 3, p. 337-44, 2008.

ARAUJO, Núbia da Silva; RODRIGUES, Cristiane Teixeira; CURY, Márcia Cristina. Helmintos em caixas de areia em creches da cidade de Uberlândia, Minas Gerais. Revista Saúde Pública, v. 42, n. 1, p. 150-3, 2008. 
ASSIS, Tália S. Machado de; FREITAS, Isabela C. Menezes de; CARVALHO, Fabiano Duarte. Avaliação da presença de ovos de helmintos intestinais em ônibus do transporte público de Contagem, Minas Gerais. Educação \& Tecnologia, v. 22, n. 2, 2018.

BEYHAN, Yunus E.; IILMAZ, Hasan; HOKELEK, Murat. Effects of acetic acid on the viability of Ascarislumbricoides eggs. Saudi Medical Journal, v. 37, n. 3, p. 288-92, 2016.

BORGES, Clarissa A.; COSTA-CRUZ, Julia M.; PAULA, Fabiana M. de. Intestinal parasites inside public restrooms and buses from the city of Uberlândia, Minas Gerais, Brazil. Revista do Instituto Medicina Tropical, v. 51, n. 4, p. 223-25, 2009.

BRITO, Fernanda Maria de; LOPES, Karla; VELHO, Nádia de Campos. Frequência de ovos de helmintos e de cistos de protozários em dinheiro. Revista Univap, v 13, n. 24, p. 10, 2006.

CAMPOS, Monica Rodrigues; VALENCIA, Luiz Iván Ortiz; FORTES, Bruno P.M. Drumond; BRAGA, Ricardo Cerqueira Campos; MEDRONHO, R.A. Distribuição espacial da infecção por Ascaris lumbricoides. Revista de Saúde Pública, v. 35, n. 1, p. 69-74, 2002.

CHEN, Anne Agnesini; MUCCl, José Luiz Negrão. Frequency of helminth contamination in child day care playgrounds in the city of Varzea Paulista, Sao Paulo, Brazil. Revista de Patologia Tropical, v. 41, n. 2, p. 195-202, 2015.

COELHO, Lina M. de Petrini da Silva; AIDAR SOBRINHO, Tuffi; OLIVEIRA, Sônia Maria de; IKEGAMI, Marcelo Tetsuo; YOSHIZUMI, Alexandre Massao; NAKAMOTO, Allyson Y. Koda et al. Ovos e larvas de helmintos nos sanitários de pré-escolas municipais de Sorocaba, SP e suas frequências nas fezes das crianças. Revista Sociedade Brasileira de Medicina Tropical, v. 2, n. 6, p. 647-52, 1999.

COOK, G.C. Enterobius vermicularis infection. Gut, v. 35, n. 9, p. 1159-62, 1994. 
CORRÊA, Lúcia Lacerda de; SILVA, Maria I.P. Gonçalves da; SILVA, Rita Maria da; DIAS, Rosa Maria Donini Souza. Ancylostoma duodenale e Necator americanus: diagnóstico diferencial das larvas infestantes e prevalência em amostras fecais provenientes da grande São Paulo. Revista Instituto Adolfo Lutz, v. 32, n. 2, p. 14553, 1979.

COSTA, Marina A.; TEODORO, Layane M.; OLIVEIRA, Gustavo H. Bahia de; NUNES, Ana Paula; BARATA, Ricardo A. Intestinal parasites in paper money circulating in the city of Diamantina (Minas Gerais, Brazil). Research and Reports in Tropical Medicine, v. 9, p. 77-80, 2018.

COSTA-CRUZ, Julia Maria; CARDOSO, Margareth L. Gennari; MARQUES, Daldy Endo. Intestinal parasites in school food handlers in the city of Uberlândia, Minas Gerais, Brazil. Revista do Instituto de Medicina Tropical, v. 37, n. 3, p. 191-6, 1995.

CURVAL, Larissa Gabrielle; FRANÇA, Adriana de Oliveira; FERNANDES, Henrique Jorge; MENDES, Rinaldo Pôncio; CARVALHO, Lídia Raquel de; MINORI, Higa Alemão et al. Prevalence of intestinal parasites among inmates in Midwest Brazil. PLoS One, v. 12, n. 9, p. e0182248, 2017.

DAMÁZIO, Schayra Minine; MACIEL, Janaina Santos; AMORIM, Renan Florindo; SOUZA, Marco Antônio A. de. Ocorrência de parasitos intestinais em cédulas de dinheiro circulantes no comércio do município de São Mateus, Espírito Santo, Brasil. Revista Brasileira de Geografia Médica e da Saúde, v. 11, n. 29, p. 12-9, 2015.

DOLD, Christina; HOLLAND, Celia V. Ascaris and ascariasis. Microbes and Infection, v. 13, n. 7, p. 632-7, 2011.

FERNANDES, Adriana A. L.; RANGEL, Carla D.; SENA, Cláudia J.C.; RANGEL, Cleber V.; MORAES, Rodrigo. Diversidade de bactérias, fungos e formas de resistência de parasitos em duas rotas de ônibus do transporte coletivo da Grande Vitória/ES. Revista Sapientia, v. 11, p. 39-45, 2012. 
GOMES, Tiago Martins; SILVA, José O. Rosa da; CARDOSO, Sandra R. Afonso. Pesquisa de enteroparasitas em meios de transporte público urbano da cidade de Patos de Minas, MG, Brasil. Psicologia e Saúde em Debate, v. 2, n. 1, p. 74-99, 2016.

GUILHERME, Ana Lucia Falavigna; ARAÚJO, Silvana Marques de; FALAVIGNA, Dina Lúcia Morais; PUPULIM, Áurea Regina Teles; DIAS, Maria Luiza G. Goulart; OLIVEIRA, Henrique Sérgio de et al. Prevalência de enteroparasitas em horticultores e hortaliças da Feira do Produtor de Maringá, Paraná. Revista da Sociedade Brasileira de Medicina Tropical, v. 32, n. 4, p. 405-11, 1999.

ISAAC, Clement; TURAY, P.N.; INEGBENOSUN, Collins U.; EZEKIEL, Solomon A.; ADAMU, H.P.; OHIOLE, J.A. Prevalence of soil-transmitted helminths in primary school playgrounds in Edo State, Southern Nigeria. Helminthologia, v. 56, n. 4, p. 282-95, 2019.

LEVAI, Eduardo Virgilio; AMATO-NETO; Vicente; CAMPOS, Rubens; PINTO, Pedro Luiz Silva; MOREIRA, Antonio Augusto Baillot; SANT'ANA, Eunice José de et al. Pesquisa de ovos de helmintos e de cistos de protozoários em dinheiro. Revista de Saúde Pública, 1986; v.20; n.1, 1986.

MATESCO, Viviana Cauduro; MENTZ, Márcia Bohrer; ROTT, Marilise Brittes; SILVEIRA, Caroline de Oliveira. Contaminação sazonal por ovos de helmintos na praia de ipanema, em Porto Alegre, Rio Grande do Sul, Brasil. Revista de Patologia Tropical, v. 35, n. 2, p. 135-42, 2007.

MENEZES, Valesca Francisco Pinto; MEDEIROS, Niara da Silva; DANI, Caroline. Prevalência de enteroparasitoses em escolares: uma revisão do perfil encontrado nas diferentes regiões do Brasil. Revista Brasileira Multidisciplinar, v. 15, n. 2, p. 7-18, 2012.

MURTA, Felipe Leão; MASSARA, Cristiano Lara. Presença de ovos de helmintos intestinais em ônibus do transporte público em Belo Horizonte. Revista de Patologia Tropical, v. 38, n. 3, p. 207-12. 2015. 
NEVES, David Pereira. Parasitologia humana. 13. ed. São Paulo: Atheneu, 2016.

NOMURA, Priscila Ruzzon; FERREIRA, Ana Rúbia Magalhães; RAFAELLI, Rafael Algusto; AUGUSTO, Jessica Grande; TATAKIHARA, Vera L. Hideko; CUSTÓDIO, Luiz Antonio et al. Estudo da incidência de parasitas intestinais em verduras comercializadas em feira livre e supermercado de Londrina. Revista Semina, v. 36, n. 1, p. 209-14, 2015.

PEDROSO, Reginaldo S.; SIQUEIRA, Rosângela V. Pesquisa de cistos de protozoários, larvas e ovos de helmintos em chupetas. Jornal de Pediatria, v. 73, n. 1, p. 21-5, 1997.

PEDROSO, Robson Francisco; AMARANTE, Marla Karine. Giardíase: aspectos parasitológicos e imunológicos. Biosaúde, v. 8, n. 1, p. 61-72, 2006.

PICCOLO, Lilian; GAGLIANI, Luiz Henrique. Estudo da prevalência de helmintos e protozoários em notas de dinheiro (papel-moeda) em circulação na baixada santista. Revista Unilus, v. 5, n. 9, p. 13-20, 2008.

POULSEN, Casper Sahl; STENSVOLD, Christen Rune. Systematic review on Endolimax nana: a less well studied intestinal ameba. Tropical Parasitology, v. 6, n. 1, p. 8-29, 2016.

REY, Luís. Bases da parasitologia médica. 3. ed. Rio de Janeiro: Guanabara Koogan, 2010.

ROSS, Marcelo; SCHMITT, Bruna Aimée Meinen; PAULA, Daiane de; TOMAZZI, Rita de Cássia; CECCHIN, Rita Stéfani; KUNH, Fernanda et al. Prevalência de ovos, larvas, cistos e oocistos de parasitas com potencial zoonótico em praças públicas e áreas de lazer na cidade de Cruz Alta/RS: análise preliminar. In: MOSTRA DE INICIAÇÃO CIENTÍFICA, XVI. Anais... Cruz Alta: UNICRUZ, 2011. 
SANTANA, Luiz Alberto; VITORINO, Rodrigo Roger; ANTONIO, Vanderson Esperidião; MOREIRA, Tiago Ricardo; GOMES, Andréia Patrícia. Atualidades sobre giardíase. Jornal Brasileiro de Medicina, v. 102, n. 1, 2014.

SANTARÉM, Vamilton Alvares; SARTOR, Izidoro Francisco; BERGAMO, Fabiana M. Matsubara. Contaminação por ovos de Toxocara spp de parques e praças públicas de Botucatu, São Paulo, Brasil. Revista da Sociedade Brasileira de Medicina Tropical, v. 31, n. 6, p. 529-32, 1998.

SANTOS, Fred L. Neves; SOARES, Neci Matos. Mecanismos fisiopatogênicos e diagnóstico laboratorial da infecção causada pela Entamoeba histolytica. Jornal Brasileiro de Patologia e Medicina Laboratorial, v. 44, n. 4, p. 249-61, 2008.

SANTOS, Milena Garcia dos. Parasitos em transportes coletivos urbanos da cidade de Cascavel, Paraná. Cascavel: Faculdade Assis Gurgacz, 2015.

SCHÄR, Fabian; TROSTDORF, Ulf; GIARDINA, Federica; KHIEU, Virak; MUTH, Sinuon; MARTI, Hanspeter et al. Strongyloides stercoralis: global distribution and risk factors. PLOS Neglected Tropical Diseases, v. 7, n. 7, p. e2288, 2013.

SCHOLTE, Ronaldo G. Carvalho; CARVALHO, Omar dos Santos; LIMA, Grécia M. Nunes de; ARAUJO, Amanda Domingues; MASSARA, Cristiano Lara. Busca de ovos de helmintos intestinais nos terminais de aeroporto Carlos Drummond de Andrade (Pampulha), Belo Horizonte e Tancredo Neves, Confins, Minas Gerais, Brasil. Revista de Patologia Tropical, v. 44, n. 3, p. 303-11, 2015.

SILVA, Alexandre Teixeira; MASSARA, Cristiano Lara; MURTA, Felipe Gomes Leão; OLIVEIRA, Áureo Almeida; SILVA, Fabiana de Oliveira Lara. Ovos de Enterobius vermicularis em salas de espera e banheiros de unidades básicas de saúde (UBS) do município de Nova Serrana-MG: contribuições para o controle. Revista de Patologia Tropical, v. 42, p. 425-33, 2013. 
SMITH, A. Marlins. Measurements of and observations upon the cysts of Entamoeba histolytica and of Entamoeba coli. Annals of Tropical Medicine \& Parasitology, v. 12, n. 1, p. 27-69, 2017.

SUDRÉ, Adriana; FRANCO, Bárbara; ZANIBONI, Bruna; GONÇALVES, Daniel; SANTOS, Fernanda; BRANCO, Letícia et al. Estudo da contaminação de moedas e cédulas de dinheiro circulantes na cidade de Niterói/RJ. Revista de Patologia Tropical, v. 41, n. 4, p. 465-70, 2012.

TOMÉ, Juliane B.S.; TAVARES, Rejane G. Diferenciação entre Entamoeba histolytica e Entamoeba dispar por meio de ensaio imunoenzimático para pesquisa de antígenos em amostras fecais. Revista do Instituto Adolfo Lutz, v. 66, n. 3, p. 305-7, 2007.

VALADARES, Mayara Batista; FONSECA, Hugo Maia; WELTER, Áurea. Parasitos intestinais em sanitários públicos da cidade de Palmas/TO. Revista Cereus, v. 6, n. 1, p. 19-34, 2014.

VALENTE, Vanderson Firmiano. Dinâmica da infecção e reinfecção por ancilostomídeos seguido ao tratamento anti-helmíntico em crianças residentes em seis comunidades dos municípios de Novo Oriente de Minas e Caraí na região nordeste de Minas Gerais, Brasil. Dissertação (mestrado em ciências) Fundação Oswaldo Cruz, 2013.

WHITE-JÚNIOR, A. Clinton; COYLE, Christina M.; RAJSHEKHAR, Vedantam; SINGH, Gagandeep; HAUSER, W. Allen; MOHANTY, Aaron et al. Diagnosis and treatment of neurocysticercosis: 2017 clinical practice guidelines by the infectious diseases society of America (IDSA) and the American society of tropical medicine and Hygiene (ASTMH). Clinical Infectious Diseases, v. 66, n. 8, p. 1159-63, 2018.

Enviado: Setembro, 2020.

Aprovado: Outubro, 2020. 